\title{
Thyroid endocrinology: the future is now
}

\section{Domenico Salvatore*}

Department of Molecular and Clinical Endocrinology and Oncology, University of Naples "Federico II," Naples, Italy

*Correspondence: domsalva@unina.it

...E quelli che s'inarnorono di pratica senza scientia sono come il nocchiere che entra navilio senza timone e bussola, e che mai ha certezza dove si vada...

[He who loves practice without theory is like the sailor who boards ship without a rudder and compass and never knows where he may be cast.] (Leonardo Da Vinci)

Thyroid endocrinology aims to advance our knowledge of the physiopathology of the thyroid gland. The thyroidologist, like all physicians concerned with internal medicine, lives in an environment of increasing specialization and multiplying subspecialties. This applies also to thyroid research, in which the divide between "basic" and "clinical" scientists is often wider than it should be. Consequently, the grand challenge that Frontiers in Thyroid Endocrinology faces is to provide a common platform for the opinions and rigorous investigations of both of them. Of course, the link will be the so-called "translational research," in which both sides of the coin merge in the ultimate interest of the patient affected by thyroid disease.

I will quote only a few examples of a nonexhaustive list of ideas and a few examples of "hot topics" that are jostling for attention. I am fully aware of the limits of this list, but my aim is to give a flavor of what and where the grand challenges are and what awaits us in the near future.

\section{CLINICAL ISSUES}

Thyroid disease and specific ages: prevalence, clinical manifestations and treatment of thyroid disorders differ greatly during the course of life. In puberty, early diagnosis and prevention of thyroid disease are of tremendous socio-economical relevance because a significant fraction of goiters develops at this age. The big exception is iodine deficiency, which represents the most common thyroid disease for all ages. In adults, iodine deficiency remains a worldwide risk that is responsible for morbidity associated with nodular goiter and thyroid autonomy. Iodine supplementation has been one of the great achievements of preventive medicine although it presents new challenges to us as a society: does iodine supplementation increase the risk of developing thyroid diseases, among which thyroid autoimmunity? (de Carvalho et al., 2009; Weetman, 2009). This question must be addressed in a scientifically rigorous fashion. During the first decade of the twentyfirst century, the population reaching 65 years of age has increased exponentially. Thyroid diseases in the elderly are often unrecognized and may result in substantial morbidity and mortality. There are a variety of diagnostic and therapeutic problems in aged individuals that are relevant not only for the endocrinologist, but also for the geriatrician and oncologist. The issue of subclinical thyroid diseases, its diagnosis and whether and how it should be treated are still open questions notwithstanding years of robust clinical studies. Finally, the thyroid nodule is still the center of interest for thousands of investigators and clinicians. Thyroid cancer is the most common form of malignant endocrine tumor. The prognosis of thyroid cancer as a whole is generally favorable representing only $0.5 \%$ of all cancer deaths. However, the prognosis for patients with metastatic, iodine nonavid, differentiated thyroid cancer, and anaplastic cancer remains poor (Boelaert, 2010). What controls the growth of thyroid epithelial cells and how this is related to genetic or environmental cues are questions that remain unanswered. Predictors of malignancy (molecules expressed, hormone levels, or genetic alterations or polymorphisms) that could bring about an early diagnosis and favorable prognosis of thyroid nodules remain a mirage. Future research in this field should learn to exploit the recent discoveries made in the realm of the "omics" technology, including molecular genomics, proteomics, and metabolomics. Thanks to their global analysis and systematic approach, these techniques will produce huge amounts of data that will be instrumental for the definition and treatment of the thyroid nodule. From a clinical point of view, some differentiated cancers are clinically "benign" and would not even require radioiodine treatment. Some others are much more aggressive and require an aggressive approach. How can we distinguish among these cancer types in order to decide the most appropriate therapeutic approach? A better understanding of the molecular pathogenesis of thyroid cancer coupled with the significant advances made in cancer therapy guarantees promising and exciting therapeutic options in years to come.

\section{TRANSLATIONAL ISSUES}

Metastatic thyroid cancer poorly sensitive to radioiodine ablation has been associated with a number of oncogenic genetic abnormalities that are unique. A clear need for new therapeutic approaches exists given the chemo- and radio-insensitivity of the majority of these tumors. The identification over the last 5-10 years of many hallmarks of thyroid cancers led to clinical trials of new "molecular" therapies for all histotypes of thyroid cancer, and in particular medullary thyroid carcinoma. A number of kinase inhibitor drugs have been shown to exert experimental activity and are currently undergoing clinical trials. These agents are mostly multi-kinase inhibitors that act mainly on RET, cMET, and VEGF, but are also able to affect other kinases involved in angiogenesis. There are multiple agents that have been tested at phase II levels with tremendous results that warrant validation in phase III clinical trials. Future trials should perhaps investigate the efficacy of combination targeted therapy in the first-line treatment of advanced thyroid cancer as well as better define their side effects, and more importantly, how to treat them (Schlumberger and Sherman, 2009; Sherman, 2010). Of course, these patients are fortunately rare, and research groups from different continents will have to join forces to gather together large cohorts of patients in order to conduct prospective 
studies. This new avenue of cancer treatment represents, in my opinion, the most exciting promise of translational thyroidology.

\section{BASIC ISSUES}

Finally, the basic science. The last 20 years of basic thyroid research generated pivotal discoveries that opened new frontiers in thyroidology. The cloning of the three iodothyronine selenodeiodinases and the identification of specific thyroid hormone transporters were breakthroughs that impacted not only on the physiology of thyroid hormone action but also on the identification of new diseases associated with their genetic abnormalities (Bianco et al., 2002; Gereben et al., 2008; Visser et al., 2008). The regulation of T3 generation at tissue level has been increasingly recognized as an important mechanism for the control thyroid hormone action. The fraction of T3 that is produced locally from T4, and the contribution of locally produced T3 to the amount of T3 bound to its receptors varies substantially from species to species and from tissue to tissue. In humans, approximately $80 \%$ of extrathyroidal T3 derived from T4 is produced intracellularly. This locally produced T3 is a crucial factor in several fundamental mechanisms, including circulating thyroid homeostasis, energy balance, and glucose metabolism. The concept that circulating thyroid hormone does not necessarily reflect the tissue or cellular thyroid hormone action opens the intriguing possibility of modifying and fine tuning at will thyroid hormone action in selected tissues. Relevant to this concept was the identification of selective and receptor-specific thyroid hormone analogs as well as a novel class of biogenic amines related to thyroid hormone called "thyronamines." These are putative metabolites of thyroid hormone that have biological activities distinct from those of thyroxine or T3, and could either play a role in the modulation of thyroid hormone action or act as endogenous regulators of other distinct pathways (Piehl et al., 2010). All this opens the exciting possibility of deliberately modifying $\mathrm{TH}$ action in a selected tissue to achieve therapeutic goals not necessarily related to thyroid itself.

We at Frontiers in Thyroid Endocrinology are pleased to take-up the challenge of becoming a platform for the publication of high quality investigations concerning all aspects of thyroid research, across all species and in all its multiple aspects, from molecular biology to clinical studies. We welcome contributions including cutting edge discoveries in the areas of thyroid physiopathology, basic related science, cellular and animal physiology, as well as, and most importantly, therapy and treatment of thyroid disorders in humans as far as they are sustained by results of rigorous and creative investigations. Timely and original reviews especially dealing with translational aspects will be also favorably considered.

\section{REFERENCES}

Bianco, A. C., Salvatore, D., Gereben, B., Berry, M. J., and Larsen, P. R. (2002). Biochemistry, cellular and molecular biology, and physiological roles of the iodothyronine selenodeiodinases. Endocr. Rev. 23, 38-89.

Boelaert, K. (2010). Thyroid gland: revised guidelines for the management of thyroid cancer. Nat. Rev. Endocrinol. 6, 185-186.

de Carvalho, J. F., Pereira, R. M., and Shoenfeld, Y. (2009). The mosaic of autoimmunity: the role of environmental factors. Front. Biosci. (Elite Ed) 1, 501-509.

Gereben, B., Zavacki,A. M., Ribich, S., Kim, B.W.,Huang, S. A., Simonides, W.S., Zeold,A., and Bianco,A.C. (2008). Cellular and molecular basis of deiodinase-regulated thyroid hormone signaling. Endocr. Rev. 29, 898-938.

Piehl, S., Hoefig, C. S., Scanlan, T. S., and Kohrle, J. (2010). Thyronamines - past, present, and future. Endocr. Rev doi: 10.1210/er. 2009-0040

Schlumberger, M., and Sherman, S. I. (2009). Clinical trials for progressive differentiated thyroid cancer: patient selection, study design, and recent advances. Thyroid 19, 1393-1400.

Sherman, S. I. (2010). Targeted therapy of thyroid cancer. Biochem. Pharmacol. 80, 592-601.

Visser, W. E., Friesema, E. C., Jansen, J., and Visser, T. J. (2008). Thyroid hormone transport in and out of cells. Trends Endocrinol. Metab. 19, 50-56.

Weetman, A. P. (2009). The genetics of autoimmune thyroid disease. Horm. Metab. Res. 41, 421-425.

Received: 20 October 2010; accepted: 12 November 2010; published online: 07 December 2010.

Citation: Salvatore D (2010) Thyroid endocrinology: the future is now. Front. Endocrin. 1:7. doi: 10.3389/ fendo.2010.00007

This article was submitted to Frontiers in Thyroid Endocrinology, a specialty of Frontiers in Endocrinology. Copyright (C) 2010 Salvatore. This is an open-access article subject to an exclusive license agreement between the authors and the Frontiers Research Foundation, which permits unrestricted use, distribution, and reproduction in any medium, provided the original authors and source are credited. 